\title{
Pathogenesis and damage targets of hypertensive kidney injury
}

\author{
Da Sun ${ }^{1}$, Jiaojiao Wang ${ }^{1}$, Wansong Shao', Juan Wang ${ }^{1}$, Li Yao', Zilong Li ${ }^{1}$, Shinichi \\ Ohno $^{2}$ \\ 'Department of Nephrology, The First Affiliated Hospital of China Medical University, \\ Shenyang 110001, Liaoning Province, China; \\ ${ }^{2}$ University of Yamanashi, 1110 Shimokato, Chuo, Yamanashi 409-3898, Japan
}

\section{BACKGROUND}

Hypertension has a high morbidity in the general population, especially in the developed countries, and is regarded as the second leading cause of chronic kidney disease (CKD) after diabetes. ${ }^{[1,2]}$ As the major target organ affected by hypertension, the involvement of kidney is usually hard to avoid and manifested by obvious vessels, glomeruli and tubulointerstitium damage. The seminal description of nephrosclerosis was first proposed in 1918. For decades, a large number of studies related to hypertensive kidney injury have focused more on the damage in the capillary tuft causing nephroangiosclerosis and hyalinosis. More recently, light was shed on other histologic aspects and molecular mechanisms involved in the promotion of renal damage with hypertension, such as the renin-angiotensin-aldosterone system (RAAS), endothelial dysfunction, podocyte injury, calcium channel activation, oxidative stress, hypoxia induced factor (HIF) overexpression and genetic and epigenetic determinants and so on. Therefore, we should wholly revisit the classic theory of hypertensive kidney injury known as nephroangiosclerosis, ranging from a benign to a malignant form.

In the present work, we aimed at providing some new insight about hypertensive kidney injury, and for a better understanding of the current and future therapeutic strategies for global CKD progression. The impact of current therapeutic interventions for the control of hypertensive kidney injury, such as endothelial glycocalyx, is also discussed to demonstrate novel targets.

\section{RENAL HEMODYNAMIC CHANGES}

Classically, it is believed that the kidney is protected from the effects of acute systemic blood pressure (BP) increase through the auto-regulatory mechanisms, namely glomerular afferent arteriole contraction due to myogenic response and "tubuleglomerular feedback". ${ }^{[3]}$

In the early stage of hypertension, although the renal arterioles are in contraction state, the contraction of the efferent arterioles is more significant than that of the afferent arterioles, so the glomerular filtration rate can still be maintained in the normal range. However, if the arterial pressure increase becomes more severe and exceeds the range of autoregulatory protection, the increased systemic BP can directly act on the glomerular capillaries, causing intra-glomerular hypertension and hyperperfusion, which in turn leads to renal arterioles hardening and vascular intima thickening, lumen stenosis, renal vascular resistance increased. Eventually, renal blood flow is further decreased, and renal perfusion volume changes from high to low. In fact, such adaptive structural changes' response to hypertension will ensure that blood vessels withstand the increase in wall stress, and play an important role in the progression of renal ischemic damage.

Intra-glomerular hypertension results in endothelial damage and glomerular hyperfiltration enhancing glomerular permeability to macromolecules, especially plasma proteins, which causes both tubular reabsorption of proteins and mesangial 
proliferation, thus leading to tubule-interstitial inflammation and glomerulosclerosis.

In addition to physical shear stress acted on blood vessels, glomerular hypertension also exerts a direct action on glomerular structures, causing signaling regulatory responses aimed to compensate. The adaptive mechanisms include proliferation of endothelial cells, changing their signaling pathways, thereby synthesizing extracellular matrix and reorienting their cytoskeleton. Mesangial cells proliferate and activate the RAAS, producing at the same time vascular permeability factors, tumor growth factor (TGF) $-\beta$ and fibronectin. ${ }^{[4]}$

\section{ROLE OF ENDOTHELIAL DYSFUNCTION}

The metabolism of vascular endothelial cells is very active, and the integrity of structure of the vascular endothelial layer plays a pivotal role in maintaining vasomotor function and permeability. Activation of endothelium by elevated systemic BP is followed by endothelial dysfunction, which finally results in endothelial disintegration if the offending stimulus lasts longer. At the end, vascular rarefaction in the capillary system results in a decrease in tissue perfusion and consequent hypoxia..$^{[5,6]}$

Here, the synthesis of availability of nitric oxide (NO) reduced due to endothelial dysfunction is thought to be a key event underlying vascular damage. In patients with CKD, complete function of endothelial cells is not only with respect to their cardiovascular morbidity and mortality but also with regard to renal disease progression. Moreover, endogenous inhibitors of $\mathrm{NO}$ synthase, such as asymmetric dimethylarginine, also influence the synthesis of $\mathrm{NO}^{\left[{ }^{[7]}\right.}$

In endothelial and vascular smooth muscle cells, activation of reactive oxygen species (ROS)-generating enzyme results in redox signaling that activates inflammation transcription factors. ROS are potent modulators of vascular contraction/dilation, which can reduce NO bioavailability, lead to lipid peroxidation, activate proinflammatory transcription factors, increase production of growth factors and induce fibrosis. ${ }^{[8]}$

Furthermore, calcium ion channels are activated following endothelial injury, and calcium ions overflowing from the endoplasmic reticulum (ER) will increase endothelial NO synthase activity and NO production in renal vascular endothelial cells. NO relax vascular smooth muscle by inhibiting the influx of $\mathrm{Ca}$ ions and stimulating extrusion mechanisms to counteract the ischemia and hypoxia due to the reduction of blood vessels.

\section{PODOCYTE PLAY A CRUCIAL ROLE IN THE PATHOGENESIS OF HYPERTENSIVE KIDNEY INJURY}

Podocytes are important intrinsic cells of the kidney. The foot processes and the slit diaphragm (SD) constitute the out layer of filtration barrier for macromolecules and contribute to the hydraulic permeability of the glomeruli. In fact, podocytes may respond to injurious stimuli in different ways. The primary pathway leading to podocyte dysfunction is effacement, consisting in a gradual simplification of the interdigitating foot process pattern until the cell looks flat and lengthened. ${ }^{[9]}$ As injury persists, podocytes either detach from basal membrane or undergo apoptosis causing denuded areas of glomerular basal membrane responsible for a marked proteinuria.

In addition to immune damage, such as membranous nephropathy, simple mechanical stress can also cause the shedding or effacement of podocytes. Therefore, the intra-glomerular pressure elevation induced by systemic hypertension is considered as another cause of podocyte injury. In our previous study, podocytes were detected from urine sediments of patients with chronic hypertension and trace proteinuria, which was seen as the direct evidence that podocyte loss is not only associated with severe renal damage in chronic hypertension, but also occurs in the early course of hypertensive renal injury. ${ }^{[10]}$

In the past, the research on the pathogenesis of podocyte injury mainly focuses on the cytoskeletal abnormality of podocytes, oxidative stress, mitochondrial dysfunction, abnormal autophagy, and apoptosis. ${ }^{[11]}$ More recently, the use of protein tracing technology has shown that the podocyte SD complex is located in the adjacent foot processes, and it is a key structure for sensing extracellular stimulation and introducing extracellular biochemical signals into the cell. With the widespread application of the "in vivo cryotechnique" (IVCT), the true morphology and immunolocalizations of original soluble components in functioning cells and tissues under different hemodynamics can be more conveniently observed. ${ }^{[12]}$ Protein molecules such as nephrin, podocin, CD2 related protein (CD2AP), nestin, zonula occludens-1 (ZO-1) and so on, that constitute podocyte-related protein complexes, have been found to be deficient or decreased in expression under the action of hypertension in animal models. ${ }^{[13,14]}$ In our previous study, significantly decreased CD2AP expression in glomeruli in the renal biopsy from the patient with chronic hypertension has confirmed the theory mentioned above again. Of interest, CD2AP not only can inhibit the apoptosis of podocytes induced by ER stress, ${ }^{[15]}$ but can also bind to a subunit of phosphoinositide 3-kinase (PI3K) 
with nephrin, and together stimulate PI3K-dependent AKT signaling and prevent bad-mediated apoptosis of podocytes in culture. ${ }^{[16]}$

Moreover, both angiotensin II (Ang II) and ROS can directly cause apoptosis and hypertrophy of podocytes. The damage of tubular basement membrane facilitates the passage of tubular-derived products into the interstitium and peritubular capillaries' spaces, thereby accelerating fibrosis and inflammation, whereas several protein casts may obstruct the urinary flow, aggravating tubulointerstitial injury. ${ }^{[17]}$

\section{CALCIUM ION CHANNEL IS THE NEW HOTSPOT}

Most recently, another research hotspot of hypertensive kidney injury is the calcium ion channel. ${ }^{[18]}$ Transient receptor pressure channel (TRPC) is a class of receptorgated, non-selective calcium cation channel proteins, which are mainly expressed in the podocyte. ${ }^{[19]}$ TRPC6 is activated and opened when podocytes are stimulated. Subsequent to calcium influx, abnormal activity of signal transduction pathways and downstream cytokines resulting from the transient change of hemodynamics.

Downstream signaling pathways, such as $\mathrm{PKA} / \mathrm{Ca} 2+$ / calmodulin-dependent protein kinase II (CaMKII) signaling, Ca2+ dependent Rho GTPase signaling, Calcineurin-Nuclear factor of activated T cell (NFAT) transcriptional activation are involved in contributing to podocyte cytoskeleton rearrangement and apoptosis under hypertension, ultimately leading to proteinuria. When TRPC6 or its upstream signal is blocked, podocyte injury can be alleviated. ${ }^{[20,21]}$

Connexin43 (Cx43) is a major component of gap junctions and hemichannels, localized on podocyte $\mathrm{SD}$, plays a unique role in intracellular communication by directly permitting substances such as calcium, ATP in-and-out of cells. ${ }^{[22]} \mathrm{It}$ has been shown that up-regulated expression of $\mathrm{Cx} 43$ and hemichannel opening in response to kidney stress induced by Ang II, followed by calcium influx and the release of ATP into extracellular fluid. ${ }^{[23]}$ CX43 may supplement TRPC6 in the regulation of calcium influx, and plays a crucial role in podocyte injury due to hypertension.

All taken together, these findings improve our understanding of the mechanism of podocyte injury during hypertension.

\section{ROLE OF RAAS}

The renal hemodynamic mechanism of Ang II is mainly manifested in its direct effect on renal vascular smooth muscle cells, causing vasoconstriction of both afferent and efferent arterioles, leading to the development of glomerular capillary hypertension and the decrease of renal blood flow. In patients with CKD, the renal local RAAS system is activated, intrarenal Ang II levels are increased respond to the amplified sensitivity of the tubuleglomerular feedback.

Ang II also has several non-hemodynamic effects involved in the pathogenesis of hypertensive CKD. As an effective pro-inflammatory agent, Ang II participates in the processes of chemotaxis, proliferation and differentiation of monocytes into macrophages, and contributes to modulate immune and inflammatory responses in endothelial, renal tubular and smooth muscle cellss. ${ }^{[2]}$ Moreover, Ang II induces both hypertrophy and proliferation of mesangial cells, and stimulates the processes of proliferation, apoptosis and collagen synthesis directly or through the expression of TGF- $\beta$, and causes tissue remodeling by increasing proliferation of interstitial fibroblasts, as well as by decreasing apoptosis of resident interstitial cells. ${ }^{[25]}$

The classical mode of Ang II-mediated actions is to bind to own receptors on the plasma membrane, which in turn phosphorylates the receptors and activates downstream signal transduction, leading to intracellular responses at the end. However, increasing evidence suggests that binding of Ang II to AT1 receptor activates endocytotic processes. Upon internalization, Ang II stimulates cytoplasmic and nuclear AT1-receptors to increase intracellular calcium, and it activates nuclear transcription factor nuclear factor- $\varkappa \mathrm{B}$, leading to increased expression of the $\mathrm{Na}^{+} / \mathrm{H}^{+}$exchanger NHE-3, pro-inflammatory cytokines and growth factors. Ang II also stimulates ROS production by inducing vascular NADPH oxidase and endothelin-1 (ET-1) expression in the kidneys. ${ }^{[22,26]}$

Apart from Ang II, other RAAS components are involved in the inflammatory and fibrotic renal damage. In particular, aldosterone exerts a pro-oxidant action mediated through different pathways, including NADPH oxidase-dependent mechanisms. The mechanism of pro-inflammatory and pro-fibrotic effects of aldosterone were mediated by nuclear factor- $x \mathrm{~B}$ activation, a factor involved in inflammation, immunity, cell proliferation and apoptosis. ${ }^{[27]}$ Furthermore, aldosterone potentiates the mitogenic activity of TGF- $\beta$, thus leading to renal fibrosis and glomerulosclerosis.

\section{PERSPECTIVES}

According to a large number of evidence-based medical studies, it is considered that RAAS inhibitors are the first-line therapy drugs for hypertensive patients with kidney disease, as they effectively reduce proteinuria and 
CKD progression, despite being almost equally effective compared with other antihypertensive drugs at reducing BP. Notably, although monotherapy with RAAS blockers results were beneficial, dual RAAS inhibition did not demonstrate to provide additional benefits in terms of renal protection, but rather led to a frequent worsening of renal function. Nevertheless, a new scenario has been recently brought to the attention of the medical community, that is, the treatment with ACEI and ARBs has the potential to increase the number of glomerular and parietal podocytes, a phenomenon contributing to glomerulosclerosis regression under ACEI therapy. ${ }^{[28]}$ In particular, remodeling of the Bowman's capsule epithelial cells appears as a key feature of ACEI or ARBs renoprotection.

On the other hand, improving the blood flow of the renal microvasculature to alleviate ischemia and hypoxia caused by the reduction of blood vessels have always been the direction of clinicians' efforts. The glycosaminoglycan (GAG) is a negatively charged reticular lining on the vascular endothelium, widely existing in humans and animals. It is connected to the endothelium through several skeleton molecules, mainly includes proteoglycan, hyaluronic acid, dermatan sulfate and heparan sulfate and so on. The glycocalyx forms a network that integrates soluble molecules from plasma and endothelium. There is a dynamic balance between this layer of soluble components and the flowing blood, which continuously affects the thickness of the glycocalyx composition. Endothelial GAG can repair vascular endothelium, and contributes to realizing a variety of protective effects such as increasing the availability of NO, reducing oxidative stress, reducing vascular permeability, inhibiting platelet adhesion, regulating coagulation factors, inhibiting leukocyte adhesion and blood cell exudation and so on. ${ }^{[29]}$

Furthermore, HIF is another hotspot in pathogenesis of hypertensive kidney injury. In recent years, there have been more in-depth studies on the therapeutic mechanism of HIF in renal anemia. Hypoxic conditions trigger mitochondrial dysfunction and can activate the transcription factor HIF, which trigger the downstream signal pathway and ultimately contribute to the tubular atrophy and interstitial fibrosis. In a previous study, HIF1 $\alpha$ gene expression in renal endothelia was induced by Ang II in a Nuclear Factor- $x$ B $(\mathrm{NF} x \mathrm{~B})$-dependent manner and increased expression of HIF $1 \alpha$ is correlated with glomerular injury and promote the progression of hypertensive CKD. ${ }^{[30]}$

The bench-to-bedside transition of insight into the molecular mechanisms of renal injury in hypertensive patients, and also a profound understanding of the molecular pathogenesis of hypertensive renal injury with other/mixed etiology, may lead to the development novel treatment approaches, such as HIF stabilization, ET1 blockade, antiinflammatory therapy or immunosuppression. There is still a long way to go for related mechanism research, but once realized, personalized medicine is expected to play a role in hypertensive kidney injury.

\section{Conflict of Interest}

None declared.

\section{REFERENCES}

1 DiMagno EP, DiMagno MJ. National Institute of Diabetes and Digestive and Kidney Diseases workshop on EUS and related technologies: history of EUS. Gastrointest Endosc 2018; 88: 205-6.

2 Rivera SL, Martin J, Landry J. Acute and Chronic Hypertension: What Clinicians Need to Know for Diagnosis and Management. Crit Care Nurs Clin North Am 2019; 31: 97-108.

3 Hill GS. Hypertensive nephrosclerosis. Curr Opin Nephrol Hypertens 2008; 17: 266-70.

4 Pavenstadt H, Kriz W, Kretzler M. Cell biology of the glomerular podocyte. Physiol Rev 2003; 83: 253-307.

5 Bai L, Shyy JYP. Shear Stress Regulation of Endothelium: A Double-edged Sword. J Transl Int Med 2018; 6: 58-61.

6 Rajendran G, Schonfeld MP, Tiwari R, Huang S, Torosyan R, Fields T, et al. Inhibition of Endothelial PHD2 Suppresses Post-Ischemic Kidney Inflammation through Hypoxia-Inducible Factor-1. J Am Soc Nephrol 2020; 31: 501-16.

7 Baylis C. Nitric oxide synthase derangements and hypertension in kidney disease. Curr Opin Nephrol Hypertens 2012; 21: 1-6.

8 Wilcox CS. Oxidative stress and nitric oxide deficiency in the kidney: a critical link to hypertension? Am J Physiol Regul Integr Comp Physiol 2005; 289: R913-35.

9 Shankland SJ. The podocyte's response to injury: role in proteinuria and glomerulosclerosis. Kidney Int 2006; 69: 2131-2147.

10 Sun D, Wang JJ, Wang W, Wang J, Wang LN, Yao L, et al. Human podocyte injury in the early course of hypertensive renal injury. World J Clin Cases 2019; 7: 3698-710.

11 Tagawa A, Yasuda M, Kume S, Yamahara K, Nakazawa J, Chin-Kanasaki M, et al. Impaired Podocyte Autophagy Exacerbates Proteinuria in Diabetic Nephropathy. Diabetes 2016; 65: 755-67.

12 Yuan X, Wang W, Wang J, Yin X, Zhai X, Wang L, et al. Down-regulation of integrin betal and focal adhesion kinase in renal glomeruli under various hemodynamic conditions. PLoS One 2014; 9: e94212.

13 Kato T, Mizuguchi N, Ito A. Characteristics of podocyte injury in malignant hypertensive nephropathy of rats (MSHRSP/Kpo strain). Biomed Res 2015; 36: 313-21.

14 Garovic VD, Wagner SJ, Petrovic LM, Gray CE, Hall P, Sugimoto H, et al. Glomerular expression of nephrin and synaptopodin, but not podocin, is decreased in kidney sections from women with preeclampsia. Nephrology, dialysis, transplantation : official publication of the European Dialysis and Transplant Association - European Renal Association 2007; 22: 1136-43.

15 He F, Chen S, Wang H, Shao N, Tian X, Jiang H, et al. Regulation of $\mathrm{CD} 2$-associated protein influences podocyte endoplasmic reticulum stress-mediated apoptosis induced by albumin overload. Gene 2011; 484: 18-25.

16 Verdoodt A, Honore PM, Jacobs R, De Waele E, Van Gorp V, De Regt J, et al. Do Statins Induce or Protect from Acute Kidney Injury and Chronic Kidney Disease: An Update Review in 2018. J Transl Int Med 2018; 6: 21-5.

17 Eddy AA. Proteinuria and interstitial injury. Nephrol Dial Transplant 2004; $19: 277-81$. 
18 Peng X, Wei C, Li HZ, Li HX, Bai SZ, Wang LN, et al. NPS2390, a Selective Calcium-sensing Receptor Antagonist Controls the Phenotypic Modulation of Hypoxic Human Pulmonary Arterial Smooth Muscle Cells by Regulating Autophagy. J Transl Int Med 2019; 7: 59-68.

19 Greka A, Mundel P. Balancing calcium signals through TRPC5 and TRPC6 in podocytes. J Am Soc Nephrol 2011; 22: 1969-80.

20 Roshanravan H, Dryer SE. ATP acting through P2Y receptors causes activation of podocyte TRPC6 channels: role of podocin and reactive oxygen species. Am J Physiol Renal Physiol 2014; 306: F1088-97.

21 Szabo T, Ambrus L, Zakany N, Balla G, Biro T. Regulation of TRPC6 ion channels in podocytes - Implications for focal segmental glomerulosclerosis and acquired forms of proteinuric diseases. Acta Physiol Hung 2015; 102: 241-51.

22 Yan Q, Gao K, Chi Y, Li K, Zhu Y, Wan Y, et al. NADPH oxidase-mediated upregulation of connexin 43 contributes to podocyte injury. Free Radic Biol Med 2012; 53: 1286-97.

23 Yang M, Wang B, Li M, Jiang B. Connexin 43 is involved in aldosteroneinduced podocyte injury. Cell Physiol Biochem 2014; 34: 1652-62.

24 Pueyo ME, Gonzalez W, Nicoletti A, Savoie F, Arnal JF, Michel JB. Angiotensin II stimulates endothelial vascular cell adhesion molecule-1 via nuclear factor-kappaB activation induced by intracellular oxidative stress. Arterioscler Thromb Vasc Biol 2000; 20: 645-51.

25 Wolf G, Butzmann U, Wenzel UO. The renin-angiotensin system and progression of renal disease: from hemodynamics to cell biology. Nephron Physiol 2003; 93: P3-13.

26 Mennuni S, Rubattu S, Pierelli G, Tocci G, Fofi C, Volpe M. Hypertension and kidneys: unraveling complex molecular mechanisms underlying hypertensive renal damage. J Hum Hypertens 2014; 28: 74-9.

27 Queisser N, Oteiza PI, Stopper H, Oli RG, Schupp N. Aldosterone induces oxidative stress, oxidative DNA damage and NF-kappaB-activation in kidney tubule cells. Mol Carcinog 2011; 50: 123-35.

28 Rizzo P, Novelli R, Benigni A, Remuzzi G. Inhibiting angiotensinconverting enzyme promotes renal repair by modulating progenitor cell activation. Pharmacol Res 2016; 108: 16-22.

29 Mitra R, O'Neil GL, Harding IC, Cheng MJ, Mensah SA, Ebong EE. Glycocalyx in Atherosclerosis-Relevant Endothelium Function and as a Therapeutic Target. Curr Atheroscler Rep 2017; 19: 63.

30 Luo R, Zhang W, Zhao C, Zhang Y, Wu H, Jin J, et al. Elevated Endothelial Hypoxia-Inducible Factor-1alpha Contributes to Glomerular Injury and Promotes Hypertensive Chronic Kidney Disease. Hypertension 2015; 66 : 75-84.

How to cite this article: Sun D, Wang J, Shao W, Wang J, Yao L, Li Z, et al. Pathogenesis and damage targets of hypertensive kidney injury. $J$ Transl Intern Med 2020; 8: 205-9. 